\title{
Some new results for BVPs of first-order nonlinear integro-differential equations of volterra type
}

Yepeng Xing $^{*}$ and Yi Fu

\author{
* Correspondence: ypxing- \\ jason@hotmail.com \\ Department of Mathematics, \\ Shanghai Normal University, \\ 200234, People's Republic of China
}

\begin{abstract}
In this work we present some new results concerning the existence of solutions for first-order nonlinear integro-differential equations with boundary value conditions. Our methods to prove the existence of solutions involve new differential inequalities and classical fixed-point theorems.

MR(2000)Subject Classification. 34D09,34D99.
\end{abstract}

Keywords: Boundary value problems, integro-differential equations, fixed-point motheds

\section{Introduction and preliminaries}

As is known, integro-differential equations find many applications in various mathematical problems, see Cordunean's book [1], Guo et al.'s book [2] and references therein for details. For the recent developments involving existence of solutions to BVPs for integro-differential equations and impulsive integro-differential equations we can refer to [3-17]. So far the main method appeared in the references to guarantee the existence of solutions is the method of upper and low solutions. Motivated by the ideas in the recent works $[18,19]$, we come up with a new approach to ensure the existence of at least one solution for certain family of first-order nonlinear integro-differential equations with periodic boundary value conditions or antiperiodic boundary value conditions. Our methods involve new differential inequalities and the classical fixed-point theory.

This paper mainly considers the existence of solutions for the following first-order nonlinear integro-differential system with periodic boundary value conditions.

$$
\left\{\begin{array}{l}
x^{\prime}=f(t, x,(K x)(t)), t \in[0,1] \\
x(0)=x(1)
\end{array}\right.
$$

and first-order integro-differential system with "non-periodic" conditions.

$$
\left\{\begin{array}{l}
x^{\prime}=f(t, x,(K x)(t)), t \in[0,1] \\
A x(0)+B x(1)=\theta
\end{array}\right.
$$

where $(K x)(t)$ denotes

$$
\left(\int_{0}^{t} k_{1}(t, s) x_{1}(s) \mathrm{d} s, \int_{0}^{t} k_{2}(t, s) x_{2}(s) \mathrm{d} s, \cdots, \int_{0}^{t} k_{n}(t, s) x_{n}(s) \mathrm{d} s\right)
$$

(c) 2011 Xing and Fu; licensee Springer. This is an Open Access article distributed under the terms of the Creative Commons Attribution License (http://creativecommons.org/licenses/by/2.0), which permits unrestricted use, distribution, and reproduction in any medium, provided the original work is properly cited. 
with $k_{i}(t, s):[0,1] \times[0,1] \rightarrow[0,+\infty)$ continuous for $i=1,2, \ldots, n ; A$ and $B$ are $n \times$ $n$ matrices with real valued elements, $\theta$ is the zero vector in $\mathbb{R}^{n}$. For $A=\left(a_{i j}\right)_{n \times n}$, we denote $\|A\|$ by $\left(\sum_{i=1}^{n} \sum_{j=1}^{n}\left|a_{i j}\right|\right)^{\frac{1}{2}}$. In what follows, we assume function $f:[0,1] \times \mathbb{R}^{n}$ $\times \mathbb{R}^{n} \rightarrow \mathbb{R}^{n}$ is continuous, and $\operatorname{det}(A+B) \neq 0$.

Noticing that $\operatorname{det}(A+B) \neq 0$, conditions $A x(0)+B x(1)=\theta$ do not include the periodic conditions $x(0)=x(1)$. Furthermore, if $A=B=I$, where $I$ denotes $n \times n$ identity matrix, then $A x(0)+B x(1)=\theta$ reduces to the so-called "anti-periodic" conditions $x(0)=$ $-x(1)$. The authors of [20-24] consider this kind of "anti-periodic" conditions for differential equations or impulsive differential equations. To the best of our knowledge it is the first article to deal with integro-differential equations with "anti-periodic" conditions so far.

We are also concerned with the following BPVP of integro-differential equations of mixed type:

$$
\left\{\begin{array}{l}
x^{\prime}=f(t, x,(K x)(t),(L x)(t)), t \in[0,1] \\
x(0)=x(1)
\end{array}\right.
$$

where function $f:[0,1] \times \mathbb{R}^{n} \times \mathbb{R}^{n} \times \mathbb{R}^{n} \rightarrow \mathbb{R}^{n}$ is continuous, $(L x)(t)$ denotes

$$
\left(\int_{0}^{1} l_{1}(t, s) x_{1}(s) \mathrm{d} s, \int_{0}^{1} l_{2}(t, s) x_{2}(s) \mathrm{d} s, \cdots, \int_{0}^{1} l_{n}(t, s) x_{n}(s) \mathrm{d} s\right)
$$

with $l_{i}(t, s):[0,1] \times[0,1] \rightarrow \mathbb{R}, i=1,2, \ldots, n$ being continuous.

This article is organized as follows. In Sect. 1 we give some preliminaries. Section 2 presents some existence theorems for PVPs (1.1), (1.3) and a couple of examples to illustrate how our newly developed results work. In Sect. 3 we focus on the existence of solutions for (1.2) and also an example is given.

In what follows, if $x, y \in \mathbb{R}^{n}$, then $\langle x, y\rangle$ denotes the usual inner product and $\|x\|$ denotes the Euclidean norm of $x$ on $\mathbb{R}^{n}$. Let

$$
C\left([0,1], \mathbb{R}^{n}\right)=\left\{x:[0,1] \rightarrow \mathbb{R}^{n}, x(t) \text { is continuous }\right\}
$$

with the norm

$$
\|x\|_{C}=\sup _{t \in[0,1]}\|x(t)\| .
$$

The following well-known fixed-point theorem will be used in the proof of Theorem 3.3.

Theorem 1.1 (Schaefer)[25]. Let $X$ be a normed space with $H: X \rightarrow X$ a compact mapping. If the set

$$
S:=\{u \in X: u=\lambda H u \text { for some } \lambda \in[0,1)\}
$$

is bounded, then $\mathrm{H}$ has at least one fixed-point.

\section{Existence results for periodic conditions}

To begin with, we consider the following periodic boundary value problem

$$
\begin{aligned}
& x^{\prime}+m(t) x=g(t, x,(K x)(t)), t \in[0,1] \\
& x(0)=x(1) ;
\end{aligned}
$$


where $g:[0,1] \times \mathbb{R}^{n} \times \mathbb{R}^{n} \rightarrow \mathbb{R}^{n}$ and $m:[0,1] \rightarrow \mathbb{R}$ are both continuous functions, with $m$ having no zeros in $[0,1]$.

Lemma 2.1. The BVP (2.1) is equivalent to the integral equation

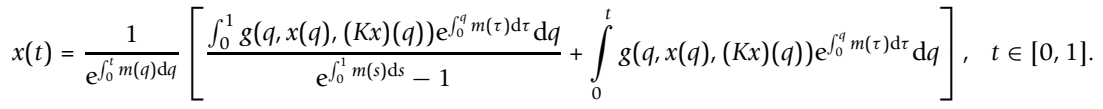

Proof. The result can be obtained by direct computation.

Theorem 2.1. Let $g$ and $m$ be as in Lemma 2.1. Assume that there exist constants $R$ $>0, \alpha \geq 0$ such that

$$
\max _{t \in[0,1]}\left[\frac{1}{\mathrm{e}^{\int_{0}^{t} m(q) \mathrm{d} q}}\left(1+\frac{1}{\left|\mathrm{e}^{\int_{0}^{1} m(q) \mathrm{d} q}-1\right|}\right)\right] M(R)<R
$$

and

$$
\begin{gathered}
\lambda\|g(t, x,(K x)(t))\| \mathrm{e}_{0}^{\int_{0}^{t} m(q) \mathrm{d} q} \leq 2 \alpha\left[\langle x, \lambda g(t, x,(K x)(t))\rangle-m(t)\|x\|^{2}\right]+M(R), \\
\forall \lambda \in[0,1] ; \forall(t, x) \in[0,1] \times B_{R}
\end{gathered}
$$

where $M(R)$ is a positive constant depending on $R, B_{R}=\left\{x \in \mathbb{R}^{n},\|x\| \leq R\right\}$. Then PBVP (2.1) has at least one solution $x \in C$ with $\|x\|_{C}<R$.

Proof. Let $C=C\left([0,1], R^{n}\right)$ and $\Omega=\left\{x(t) \in C,\|x(t)\|_{C}<R\right\}$. Define an operator $T: \bar{\Omega} \rightarrow C$ by

$$
T x(t)=\frac{1}{\mathrm{e}^{\int_{0}^{t} m(q) \mathrm{d} q}}\left[\frac{\int_{0}^{1} g(q, x(q),(K x)(q)) \mathrm{e}_{0}^{\int_{0}^{q} m(\tau) \mathrm{d} \tau} \mathrm{d} q}{\mathrm{e}^{\int_{0}^{1} m(s) \mathrm{d} s}-1}+\int_{0}^{t} g(q, x(q),(K x)(q)) \mathrm{e}^{\int_{0}^{q} m(\tau) \mathrm{d} \tau} \mathrm{d} q\right]
$$

for all $t \in[0,1]$.

Since $g$ is continuous, see that $T$ is also a continuous map. It is easy to verify the operator $T$ is compact by the Arzela-Ascoli theorem. Indeed, for the ball $\Omega$,

$$
x \neq \lambda T x, \quad \forall x \in C \text { with } x \in \partial \Omega, \quad \forall \lambda \in[0,1],
$$

implies

$$
0 \notin(I-\lambda T)(x), \forall x \in \partial \Omega, \quad \forall \lambda \in[0,1] .
$$

Define $H_{\lambda}=I-\lambda T, \lambda \in[0,1]$, where $I$ is the identity. So if (2.5) is true, then from the homotopy principle of Schauder degree [[25], Chap.4.], we have

$$
\begin{aligned}
\operatorname{deg}_{\mathrm{LS}}\left(H_{\lambda}, \Omega, 0\right) & =\operatorname{deg}_{\mathrm{LS}}(I-\lambda T, \Omega, 0) \\
& =\operatorname{deg}_{\mathrm{LS}}\left(H_{1}, \Omega, 0\right)=\operatorname{deg}_{\mathrm{LS}}\left(H_{0}, \Omega, 0\right) \\
& =\operatorname{deg}_{\mathrm{LS}}(I, \Omega, 0)=1 \neq 0 .
\end{aligned}
$$

Therefore, it follows from the non-zero property of Leray-Schauder degree that $H_{1}(x)$ $=x-T x=0$ has at least one $x \in \Omega$.

Now our problem is reduced to prove that (2.5) is true. Observe that the family of problems

$$
x=\lambda T x, \lambda \in[0,1]
$$


is equivalent to the family of PBVPs

$$
\begin{aligned}
& x^{\prime}+m(t) x=\lambda g(t, x,(K x)(t)), t \in[0,1] \\
& x(0)=x(1) .
\end{aligned}
$$

Consider the function $r(t)=\|x(t)\|^{2}, t \in[0,1]$, where $x(t)$ is a solution of (2.7). Then $r(t)$ is differentiable and we have by the product rule

$$
r^{\prime}(t)=2\left\langle x(t), x^{\prime}(t)\right\rangle=2\langle x(t), \lambda g(t, x(t),(K x)(t))-m(t) x(t)\rangle, \quad t \in[0,1] .
$$

Denote

$$
H(t)=\left[\frac{1}{\mathrm{e}^{\int_{0}^{t} m(q) \mathrm{d} q}}\left(1+\frac{1}{\left|\mathrm{e}^{\int_{0}^{1} m(q) \mathrm{d} q}-1\right|}\right)\right], \quad t \in[0,1] .
$$

Let $x$ be a solution of (2.6) with $x \in \bar{\Omega}$ We now show that $x \notin \partial \Omega$. From (2.5) and (2.3) we have, for each $t \in[0,1]$ and each $\lambda \in[0,1]$,

$$
\begin{aligned}
\|x(t)\| & =\|\lambda T x(t)\| \\
& =\frac{1}{e^{\int_{0}^{t} m(q) \mathrm{d} q}}\left[\frac{\int_{0}^{1} \lambda g(q, x(q),(K x)(q)) \int^{\int_{0}^{q} m(\tau) \mathrm{d} \tau} \mathrm{d} q}{\left|\mathrm{e}_{0}^{\int_{0}^{1} m(s) \mathrm{d} d}-1\right|}+\int_{0}^{t} \lambda g(q, x(q),(K x)(q)) \mathrm{e}^{\int_{0}^{q} m(\tau) \mathrm{d} \tau} \mathrm{d} q\right] \\
& \leq \frac{1}{\mathrm{e}_{0}^{\int_{0}^{t} m(q) \mathrm{d} q}}\left[\frac{\int_{0}^{1} \lambda\|g(q, x(q),(K x)(q))\| \mathrm{e}_{0}^{f_{0}^{q} m(\tau) \mathrm{d} \tau} \mathrm{d} q}{\left|\mathrm{e}_{0}^{\int_{0}^{1} m(s) \mathrm{d} s}-1\right|}+\int_{0}^{t} \lambda\|g(q, x(q),(K x)(q))\| \mathrm{e}^{\int_{0}^{q} m(\tau) \mathrm{d} \tau} \mathrm{d} q\right] \\
& \leq\left[\frac{1}{\mathrm{e}_{0}^{\int_{0}^{t} m(q) \mathrm{d} q}}\left(1+\frac{1}{\left|\mathrm{e}_{0}^{\int_{0}^{1} m(q) \mathrm{d} q}-1\right|}\right)\right] \int_{0}^{1} \lambda\|g(q, x(q),(K x)(q))\| \mathrm{e}_{0}^{\int_{0}^{q} m(\tau) \mathrm{d} \tau} \mathrm{d} q \\
& \leq H(t) \int_{0}^{1}\left[2 \alpha\left\langle x, \lambda g(q, x(q),(K x)(q))-m(q)\|x(q)\|^{2}\right\rangle+M(R)\right] \mathrm{d} q \\
& =H(t) \int_{0}^{1}\left[\alpha \frac{d}{\mathrm{~d} q}\left(\|x(q)\|^{2}\right)+M(R)\right] \mathrm{d} q \\
& =H(t)\left[\alpha\left(\|x(1)\|^{2}-\|x(0)\|^{2}\right)+M(R)\right] \\
& =H(t) M(R) .
\end{aligned}
$$

Then it follows from (2.2) that $x \notin \partial \Omega$. Thus, (2.5) is true and the proof is completed.

Corollary 2.1. Let $g$ and $m$ be as in Lemma 2.1 with $m(t)<0, t \in[0,1]$. If there exist constants $R>0, \alpha \geq 0$ such that

$$
\max _{t \in[0,1]}\left[\frac{1}{\mathrm{e}^{\int_{0}^{t} m(q) \mathrm{d} q}}\left(1+\frac{1}{1-\mathrm{e}^{\int_{0}^{1} m(q) \mathrm{d} q}}\right)\right] M(R)<R
$$

and

$$
\begin{gathered}
\|g(t, x,(K x)(t))\| \mathrm{e}^{\int_{0}^{t} m(q) \mathrm{d} q} \leq 2 \alpha\langle x, g(t, x,(K x)(t))\rangle+M(R), \\
\forall(t, x) \in[0,1] \times B_{R},
\end{gathered}
$$

where $M(R)$ is a positive constant depending on $R, B_{R}=\left\{x \in \mathbb{R}^{n},\|x\| \leq R\right\}$, then PBVP (2.1) has at least one solution $x \in C$ with $\|x\|_{C}<R$.

Proof. Multiply both sides of (2.8) by $\lambda \in[0,1]$ to obtain

$$
\begin{aligned}
\lambda\|g(t, x,(K x)(t))\| \mathrm{e}^{\int_{0}^{t} m(q) \mathrm{d} q} & \leq 2 \alpha\langle x, \lambda g(t, x,(K x)(t))\rangle+\lambda M(R) \\
& \leq 2 \alpha[\langle x, \lambda g(t, x,(K x)(t))\rangle]+M(R), \\
\forall(t, x) & \in[0,1] \times B_{R} .
\end{aligned}
$$


It completes the proof.

Now consider the existence of solutions of PBVP (1.1). It is easy to see (1.1) is equivalent to the PBVP

$$
\begin{aligned}
& x^{\prime}-x=f(t, x,(K x)(t))-x, t \in[0,1] \\
& x(0)=x(1) .
\end{aligned}
$$

Theorem 2.2. If there exist constants $R>0, \alpha \geq 0$ such that

$$
\frac{e(2 e-1) M(R)}{e-1}<R
$$

and

$$
\begin{aligned}
\lambda\|f(t, x,(K x)(t))-x\| e^{-t} \leq 2 \alpha\left[\langle x, \lambda f(t, x,(K x)(t))\rangle+(1-\lambda)\|x\|^{2}\right]+M(R), \\
\forall \lambda \in[0,1] ; \forall(t, x) \in[0,1] \times B_{R},
\end{aligned}
$$

where $M(R)$ is a positive constant dependent on $R, B_{R}=\left\{x \in \mathbb{R}^{n},\|x\| \leq R\right\}$, then PBVP (1.1) has at least one solution $x \in C$ with $\|x\|_{C}<R$.

Proof. Consider the PVPB (2.9), which is of the form (2.1) with $m(t) \equiv-1$ and $g(t, x$, $(K x)(t))=f(t, x,(K x)(t))-x$. Clearly,

$$
\max _{t \in[0,1]}\left[\frac{1}{\mathrm{e}^{-t}}\left(1+\frac{1}{\left|1-\mathrm{e}^{-1}\right|}\right)\right]=\frac{e(2 e-1)}{e-1} .
$$

So, (2.2) reduces to (2.10). Besides, (2.3) reduces to (2.11). Hence the result follows from Theorem 2.1.

Corollary 2.2. Assume there are constants $R>0, \alpha \geq 0$ such that

$$
\frac{e(2 e-1) M(R)}{e-1}<R
$$

and

$$
\begin{aligned}
\|f(t, x,(K x)(t))-x\| e^{-t} & \leq 2 \alpha\langle x, f(t, x,(K x)(t))\rangle+M(R), \\
\forall(t, x) & \in[0,1] \times B_{R},
\end{aligned}
$$

where $M(R)$ is a positive constant dependent on $R, B_{R}=\left\{x \in \mathbb{R}^{n},\|x\| \leq R\right\}$. Then PBVP (1.1) has at least one solution $x \in C$ with $\|x\|_{C}<R$.

Proof. Multiply both sides of $(2.13)$ by $\lambda \in[0,1]$ to obtain

$$
\begin{aligned}
\lambda\|f(t, x,(K x)(t))-x\| & \leq 2 \alpha\left[\langle x, \lambda f(t, x,(K x)(t))\rangle-\lambda\|x\|^{2}\right]+\lambda M(R) \\
& \leq 2 \alpha\left[\langle x, \lambda f(t, x,(K x)(t))\rangle+(1-\lambda)\|x\|^{2}\right]+M(R) .
\end{aligned}
$$

Considering that

$$
\lambda\|f(t, x,(K x)(t))-x\| e^{-t} \leq \lambda\|f(t, x,(K x)(t))-x\|, \forall \lambda \in[0,1] ; \forall(t, x) \in[0,1] \times B_{R},
$$

we have (2.11) is true if (2.13) is true. Then the proof is completed.

Now an example is provided to show how our theorems work. 


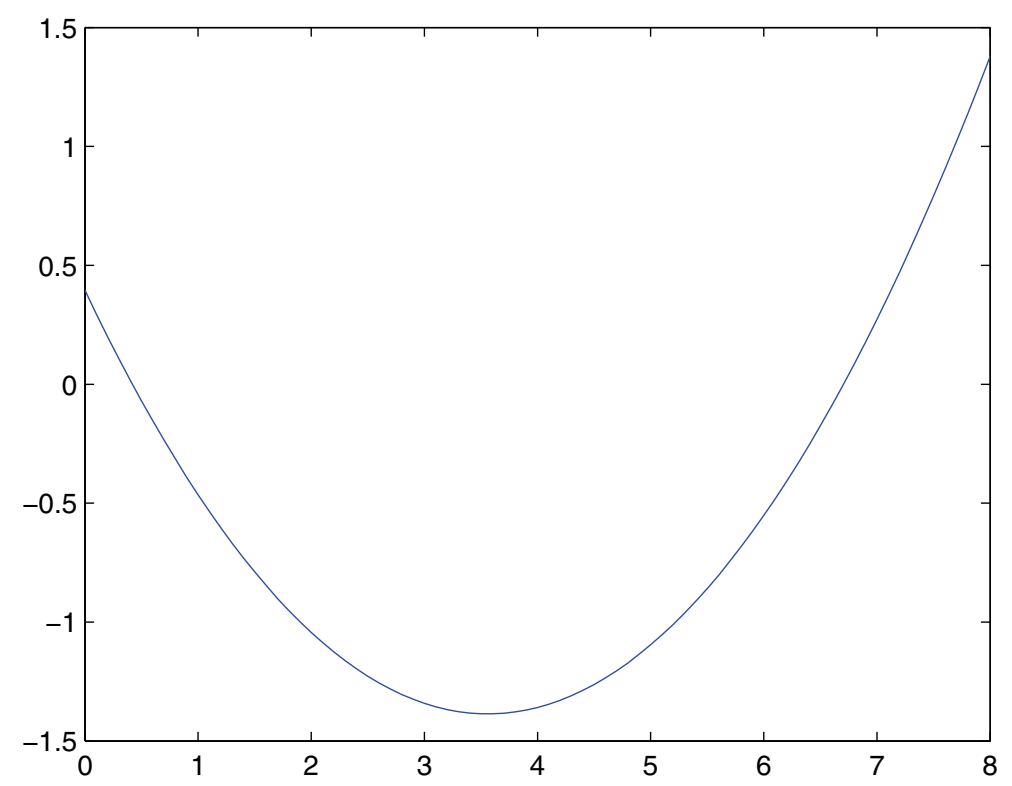

Figure 1 Figure of Example 2.1

Example 2.1 1. Consider the following PBVP with $n=2$.

$$
\left\{\begin{array}{l}
x^{\prime}=x+x y^{2}+\frac{t}{20} \\
y^{\prime}=y+\frac{y}{100} \int_{0}^{t}(t-s) x(s) \mathrm{d} s \\
x(0)=x(1), y(0)=y(1) .
\end{array}\right.
$$

Let us show (2.14) has at least one solution $(x(t), y(t))^{\top}$ with $\sqrt{x(t)^{2}+y(t)^{2}}<\frac{1}{2}, \forall t \in$ $[0,1]$.

It is clear that (2.14) has no constant solution. Let $u=(x, y)^{\top},\|u\|=\sqrt{x^{2}+y^{2}}$ and $F(t, u,(K u)(t))=\left(x+x y^{2}+\frac{t}{20}, y+\frac{y}{100} \int_{0}^{t}(t-s) x(s) \mathrm{d} s\right)^{\top}$. First note that for $\forall(t, u) \in[0,1] \times B_{R},\left|\int_{0}^{t}(t-s) x(s) \mathrm{d} s\right| \leq R$.

Then

$$
\begin{aligned}
\|F(t, u,(K u)(t))-u\| & \left.=\|\left(x y^{2}+\frac{t}{20}, \frac{y}{100} \int_{0}^{t}(t-s) x(s) \mathrm{d} s\right)^{\top}\right) \| \\
& =\sqrt{x^{2} \gamma^{4}+\frac{x y^{2} t}{10}+\frac{t^{2}}{400}+\left(\frac{y}{100} \int_{0}^{t}(t-s) x(s) \mathrm{d} s\right)^{2}} \\
& \leq \sqrt{x^{2} \gamma^{4}+\frac{|x| y^{2}}{10}+\frac{1}{400}+\left(\frac{y R}{100}\right)^{2}} \\
& \leq \sqrt{x^{2} \gamma^{4}+\frac{|x| y^{2}}{10}+\frac{1}{400}}+\sqrt{\left(\frac{y R}{100}\right)^{2}} \\
& \leq|x| y^{2}+\frac{1}{20}+\frac{R^{2}}{100}, \quad \forall(t, u) \in[0,1] \times B_{R} .
\end{aligned}
$$


On the other hand,

$$
\begin{aligned}
2 \alpha\langle u, F(t, u,(K u)(t))\rangle & =2 \alpha\left(x^{2}+x^{2} y^{2}+\frac{x t}{20}+y^{2}+\frac{y^{2}}{100} \int_{0}^{t}(t-s) x(s) \mathrm{d} s\right) \\
& \geq 2 \alpha\left(x^{2}+x^{2} y^{2}-\frac{|x|}{20}+y^{2}-\frac{y^{2} R}{100}\right) \\
& =10 x^{2}+10 x^{2} y^{2}-\frac{|x|}{2}+10 y^{2}-\frac{y^{2} R}{10}, \text { for } \alpha=5 .
\end{aligned}
$$

Clearly,

$$
\begin{gathered}
\min _{x \in \mathbb{R}}\left\{10 x^{2}-\frac{|x|}{2}\right\}=-\frac{1}{160} ; \\
10 x^{2} y^{2}+y^{2} \geq|x| y^{2}, \forall(x, y)^{\top} \in \mathbb{R}^{2} ; \\
9 y^{2}-\frac{y^{2} R}{10} \geq 0, \quad \text { if } R \leq 90 .
\end{gathered}
$$

So for some $R \leq 90$ and $\alpha=5$, we have

$$
\|F(t, u,(K u)(t))-u\| \leq 2 \alpha\langle u, F(t, u,(K u)(t))\rangle+M(R),
$$

where $M(R)=\frac{R^{2}}{50}+\frac{9}{160}$. Now it is sufficient to find a positive constant $R$ satisfying

$$
\left\{\begin{array}{l}
\frac{e(2 e-1)}{e-1} M(R)-R<0 \\
R \leq 90 .
\end{array}\right.
$$

It is easy to see that any number in $\left[\frac{1}{2}, 6\right]$ satisfies $(2.15)$. Then our conclusion follows from Corollary 2.2.

In what follows we focus on the first-order integro-differential equations of mixed type in the form of (1.2). The results presented in the following three statements are similar to Theorem 2.1, Theorem 2.2 and Corollary 2.2, respectively. So we omit all the proofs here.

Consider the following periodic boundary value problem

$$
\begin{aligned}
& x^{\prime}+m(t) x=g(t, x,(K x)(t),(L x)(t)), t \in[0,1] \\
& x(0)=x(1) ;
\end{aligned}
$$

where $g:[0,1] \times \mathbb{R}^{n} \times \mathbb{R}^{n} \times \mathbb{R}^{n} \rightarrow \mathbb{R}^{n}$ and $m:[0,1] \rightarrow \mathbb{R}$ are both continuous functions, with $m$ having no zeros in $[0,1]$.

Theorem 2.3. Assume there are constants $R>0, \alpha \geq 0$ such that

$$
\max _{t \in[0,1]}\left[\frac{1}{e^{\int_{0}^{t} m(q) \mathrm{d} q}}\left(1+\frac{1}{\left|\mathrm{e}^{\int_{0}^{1} m(q) \mathrm{d} q}-1\right|}\right)\right] M(R)<R
$$

and

$$
\begin{gathered}
\left.\lambda\|g(t, x,(K x)(t),(L x)(t))\| \int^{f_{0}^{t} m(q) \mathrm{d} q} \leq 2 \alpha[\langle x, \lambda g(t, x,(K x)(t),(L x)(t)))\rangle-m(t)\|x\|^{2}\right]+M(R), \\
\forall \lambda \in[0,1] ; \forall(t, x) \in[0,1] \times B_{R},
\end{gathered}
$$

where $M(R)$ is a positive constant depending on $R, B_{R}=\left\{x \in \mathbb{R}^{n},\|x\| \leq R\right\}$. Then PBVP (2.16) has at least one solution $x \in C$ with $\|x\|_{C}<R$.

Theorem 2.4. Suppose there are constants $R>0, \alpha \geq 0$ such that

$$
\frac{e(2 e-1) M(R)}{e-1}<R
$$


and

$$
\begin{gathered}
\lambda\|f(t, x,(K x)(t),(L x)(t))-x\| e^{-t} \leq 2 \alpha\left[\langle x, \lambda f(t, x,(K x)(t),(L x)(t))\rangle+(1-\lambda)\|x\|^{2}\right]+M(R), \\
\forall \lambda \in[0,1] ; \forall(t, x) \in[0,1] \times B_{R}
\end{gathered}
$$

where $M(R)$ is a positive constant depending on $R, B_{R}=\left\{x \in \mathbb{R}^{n},\|x\| \leq R\right\}$. Then $\operatorname{PBVP}(1.2)$ has at least one solution $x \in C$ with $\|x\|_{C}<R$.

Corollary 2.3. If there exist constants $R>0, \alpha \geq 0$ such that

$$
\frac{e(2 e-1) M(R)}{e-1}<R
$$

and

$$
\begin{aligned}
\|f(t, x,(K x)(t),(L x)(t))-x\| e^{-t} & \leq 2 \alpha\langle x, f(t, x,(K x)(t),(L x)(t))\rangle+M(R), \\
\forall(t, x) & \in[0,1] \times B_{R}
\end{aligned}
$$

where $M(R)$ is a positive constant dependent on $R, B_{R}=\left\{x \in \mathbb{R}^{n},\|x\| \leq R\right\}$, then $\operatorname{PBVP}(1.3)$ has at least one solution $x \in C$ with $\|x\|_{C}<R$.

Now we give an example to illustrate how to apply our theorems.

Example 2.2 2. Consider the following PBVP with $n=2$.

$$
\left\{\begin{array}{l}
x^{\prime}=2 x+\frac{y}{320}\left[\int_{0}^{t}(t-s) x(s) \mathrm{d} s\right]^{2} \\
y^{\prime}=3 y+\frac{x}{240} \int_{0}^{1} \mathrm{e}^{-t s} y(s) \mathrm{d} s+\frac{\cos (2 \pi t)}{360} \\
x(0)=x(1), y(0)=y(1)
\end{array}\right.
$$

We prove that (2.19) has at least one solution $(x(t), y(t))^{\top}$ with $\sqrt{x(t)^{2}+y(t)^{2}}<0.8$, $\forall t \in[0,1]$.

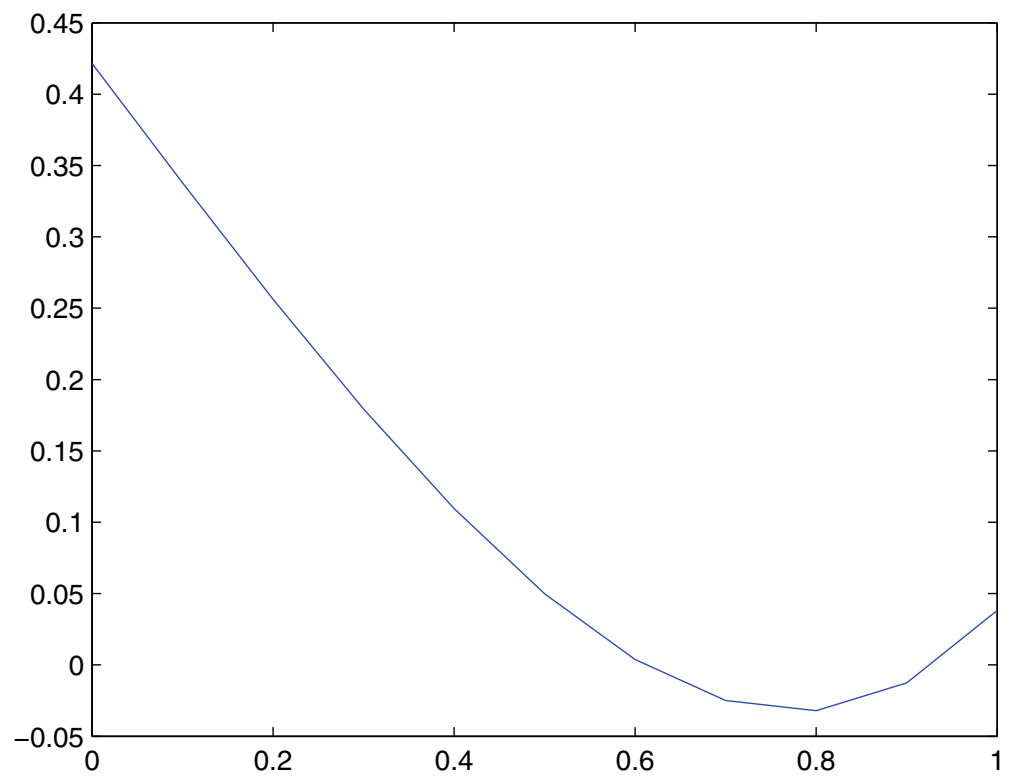


First note that (2.19) has no constant solution. Let $u=(x, y)^{\top},\|u\|=\sqrt{x^{2}+y^{2}}$ and $F(t, u,(K u)(t),(L u)(t))=\left(2 x+\frac{y}{320}\left[\int_{0}^{t}(t-s) x(s) \mathrm{d} s\right]^{2}, 3 y+\frac{x}{240} \int_{0}^{1} \mathrm{e}^{-t s} y(s) \mathrm{d} s+\frac{\cos (2 \pi t)}{360}\right)^{\mathrm{T}}$.

Since $\forall(t, u) \in[0,1] \times B_{R},\left|\frac{y}{320}\left(\int_{0}^{t}(t-s) x(s) \mathrm{d} s\right)^{2}\right| \leq \frac{R^{3}}{320}$ and $\left|\frac{x}{240} \int_{0}^{1} \mathrm{e}^{-t s} y(s) \mathrm{d} s\right| \leq \frac{R^{2}}{240}$, we obtain

$$
\begin{aligned}
\|F(t, u,(K u)(t))-u\| & \left.=\|\left(x+\frac{y}{320}\left[\int_{0}^{t}(t-s) x(s) \mathrm{d} s\right]^{2}, 2 y+\frac{x}{240} \int_{0}^{1} \mathrm{e}^{-t s} y(s) \mathrm{d} s+\frac{\cos (2 \pi t)}{360}\right)^{\top}\right) \| \\
& \leq\left|x+\frac{y}{320}\left[\int_{0}^{t}(t-s) x(s) \mathrm{d} s\right]^{2}\right|+\left|2 \gamma+\frac{x}{240} \int_{0}^{1} \mathrm{e}^{-t s} y(s) \mathrm{d} s+\frac{\cos (2 \pi t)}{360}\right| \\
& \leq|x|+\frac{R^{3}}{320}+2|y|+\frac{R^{2}}{240}+\frac{1}{360}, \quad \forall(t, u) \in[0,1] \times B_{R} .
\end{aligned}
$$

On the other hand,

$$
\begin{aligned}
& 2 \alpha\langle u, F(t, u,(K u)(t),(L u)(t))\rangle \\
& =2 \alpha\left\{2 x^{2}+\frac{x y}{320}\left[\int_{0}^{t}(t-s) x(s) \mathrm{d} s\right]^{2}+3 y^{2}+\frac{x y}{240} \int_{0}^{1} \mathrm{e}^{-t s} y(s) \mathrm{d} s+\frac{y \cos (2 \pi t)}{360}\right\} \\
& \geq 2 \alpha\left(2 x^{2}+3 y^{2}-\frac{R^{4}}{320}-\frac{R^{3}}{240}-\frac{R}{360}\right) \\
& =16 x^{2}+24 y^{2}-\frac{R^{4}}{40}-\frac{R^{3}}{30}-\frac{R}{45}, \text { for } \alpha=4 .
\end{aligned}
$$

Clearly,

$$
\begin{aligned}
& \min _{x \in \mathbb{R}}\left\{16 x^{2}-|x|\right\}=-\frac{1}{64} \\
& \min _{x \in \mathbb{R}}\left\{24 y^{2}-2|y|\right\}=-\frac{1}{24} .
\end{aligned}
$$

Thus,

$$
\|F(t, u,(K u)(t))-u\| \leq 2 \alpha\langle u, F(t, u,(K u)(t))\rangle+M(R),
$$

where

$$
M(R)=\frac{R^{4}}{40}+\frac{R^{3}}{30}+\frac{R}{45}+\frac{R^{3}}{320}+\frac{R^{2}}{240}+\frac{1}{64}+\frac{1}{360}+\frac{1}{24} .
$$

Now it is sufficient to find a positive constant $R$ satisfying

$$
\frac{e(2 e-1)}{e-1} M(R)-R<0 .
$$

We compute directly $\frac{e(2 e-1)}{e-1} M(0.8)-0.8<0$. Then our conclusion follows from Corollary 2.3.

Notice that the conclusion of Theorem 2.1 still holds if (2.3) is replaced by

$$
\begin{gathered}
\lambda\|g(t, x,(K x)(t))\| \mathrm{e}^{\int_{0}^{t} m(q) \mathrm{d} q} \leq-2 \alpha\left[\langle x, \lambda g(t, x,(K x)(t))\rangle-m(t)\|x\|^{2}\right]+M(R), \\
\forall \lambda \in[0,1] ; \forall(t, x) \in[0,1] \times B_{R} .
\end{gathered}
$$

Now we modify Theorem 2.1 and Corollary 2.2 to obtain some new results. 
Theorem 2.5. Let $g$ and $m$ be as in Lemma 2.1. Assume there exist constants $R>0$, $\alpha \geq 0$ such that

$$
\max _{t \in[0,1]}\left[\frac{1}{\mathrm{e}^{\int_{0}^{t} m(q) \mathrm{d} q}}\left(1+\frac{1}{\left|\mathrm{e}^{\int_{0}^{1} m(q) \mathrm{d} q}-1\right|}\right)\right] M(R)<R
$$

and

$$
\begin{gathered}
\lambda\|g(t, x,(K x)(t))\| \mathrm{e}^{\int_{0}^{t} m(q) \mathrm{d} q} \leq-2 \alpha\left[\langle x, \lambda g(t, x,(K x)(t))\rangle-m(t)\|x\|^{2}\right]+M(R) \\
\forall \lambda \in[0,1] ; \forall(t, x) \in[0,1] \times B_{R}
\end{gathered}
$$

where $M(R)$ is a positive constant dependent on $R, B_{R}=\left\{x \in \mathbb{R}^{n},\|x\| \leq R\right\}$. Then PBVP (2.1) has at least one solution $x \in C$ with $\|x\|_{C}<R$.

Proof. The proof is similar to that of Theorem 2.1 except choosing $r(t)=-\|x(t)\|^{2}$ instead.

See that (1.1) is equivalent to the PBVP

$$
\begin{aligned}
& x^{\prime}+x=f(t, x,(K x)(t))+x, t \in[0,1] \\
& x(0)=x(1) .
\end{aligned}
$$

Corollary 2.4. Suppose there exist constants $R>0, \alpha \geq 0$ such that

$$
\frac{e}{e-1} M(R)<R
$$

and

$$
\begin{gathered}
\|f(t, x,(K x)(t))+x\| e^{t} \leq-2 \alpha[\langle x, f(t, x,(K x)(t))\rangle]+M(R), \\
\forall \lambda \in[0,1] ; \forall(t, x) \in[0,1] \times B_{R}
\end{gathered}
$$

where $M(R)$ is a positive constant depending on $R, B_{R}=\left\{x \in \mathbb{R}^{n},\|x\| \leq R\right\}$. Then PBVP (1.1) has at least one solution $x \in C$ with $\|x\|_{C}<R$.

Proof. Consider PVPB (2.21), which is in the form from (2.1) with $m(t) \equiv 1$ and $g(t$, $x,(K x)(t))=f(t, x,(K x)(t))+x$. Clearly,

$$
\max _{t \in[0,1]}\left[\frac{1}{\mathrm{e}^{t}}\left(1+\frac{1}{|1-e|}\right)\right]=\frac{e}{e-1} .
$$

Multiply both sides of $(2.22)$ by $\lambda \in[0,1]$ to obtain

$$
\begin{aligned}
\lambda\|f(t, x,(K x)(t))+x\| e^{t} \leq & -2 \alpha[\langle x, \lambda f(t, x,(K x)(t))\rangle]+\lambda M(R) \\
\leq & -2 \alpha\left[\langle x, \lambda f(t, x,(K x)(t))\rangle+(\lambda-1)\|x\|^{2}\right]+M(R) \\
= & -2 \alpha\left[\langle x, \lambda(f(t, x,(K x)(t))+x)\rangle-\|x\|^{2}\right]+M(R), \\
& \forall(t, x) \in[0,1] \times B_{R} .
\end{aligned}
$$

Then the conclusion follows from Theorem 2.5.

Remark 2.1. Corollary 2.4 and Corollary 2.2 differ in sense that Corollary 2.4 may apply to certain problems, whereas Corollary 2.2 may not apply, and vice-versa.

Example 2.3 3. Let us prove that the PBVP

$$
\left\{\begin{array}{l}
x^{\prime}=-2 x+t x^{2}-x^{3}+\frac{1}{600}\left[-t+\int_{0}^{t} \mathrm{e}^{-t s} x(s) \mathrm{d} s\right]^{3}, \\
x(0)=x(1) .
\end{array}\right.
$$




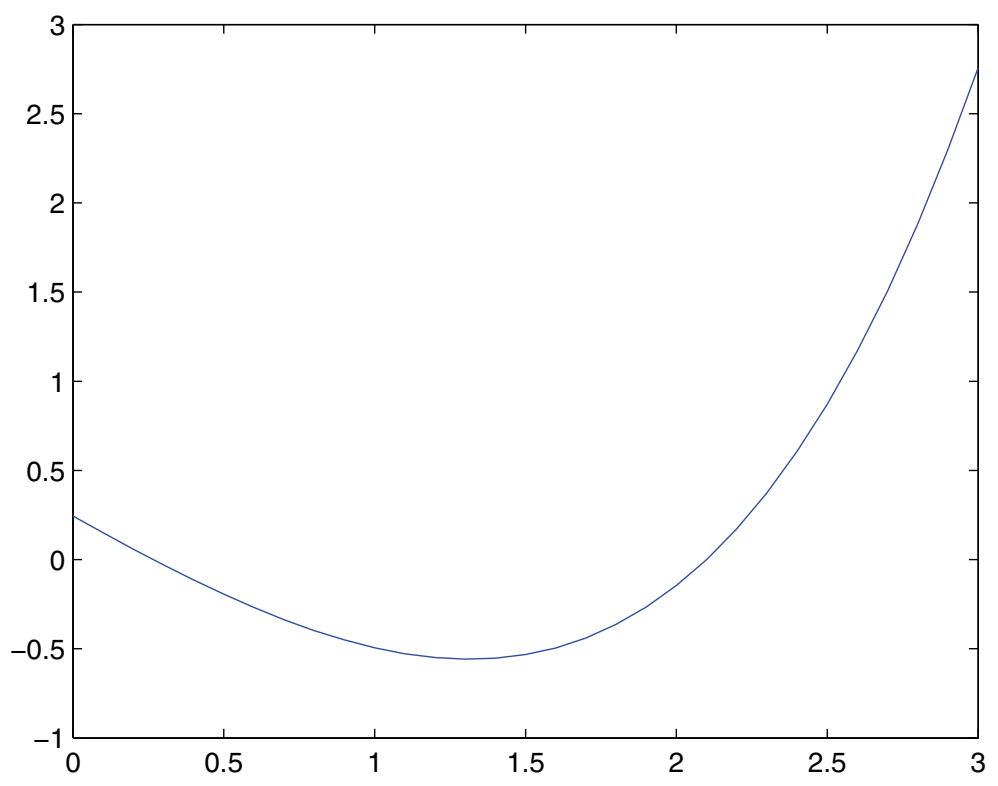

Figure 3 Figure of Example 2.3

has at least one solution $x(t)$ with $|x(t)|<1, \forall t \in[0,1]$.

Denote $f(t, x,(K x)(t))=-2 x+t x^{2}-x^{3}+\frac{1}{600}\left[-t+\int_{0}^{t} e^{-t s} x(s) \mathrm{d} s\right]^{3}$. It is clearly that for all $(t, x) \in[0,1] \times B_{R}$,

$$
\left|\frac{1}{600}\left[-t+\int_{0}^{t} e^{-t s} x(s) \mathrm{d} s\right]\right| \leq \frac{(1+R)^{3}}{600} ;
$$

Then for all $(t, x) \in[0,1] \times B_{R}$,

$$
e^{t}|f(t, x,(K x)(t))+x| \leq e\left(|x|+|x|^{2}+|x|^{3}+\frac{(1+R)^{3}}{600}\right) .
$$

On the other hand,

$$
\begin{aligned}
& -2 \alpha\langle x, f(t, x,(K x)(t))\rangle \\
& =-2 \alpha\left(-2 x+t x^{2}-x^{3}+\frac{1}{600}\left[-t+\int_{0}^{t} \mathrm{e}^{-t s} x(s) \mathrm{d} s\right]^{3}\right) \\
& =20 x^{2}+10 x^{4}-10 t x^{3}-\frac{x}{60}\left[-t+\int_{0}^{t} \mathrm{e}^{-t s} x(s) \mathrm{d} s\right]^{3}, \text { for } \alpha=5 \\
& \geq 20 x^{2}+10 x^{4}-10|x|^{3}-\frac{1}{60} R[1+R]^{3} .
\end{aligned}
$$

Taking into account that

$$
\min _{x \in \mathbb{R}}\left\{20 x^{2}+10 x^{4}-10|x|^{3}-e\left(|x|+|x|^{2}+|x|^{3}\right)\right\} \geq-0.15
$$


we choose

$$
M(R)=\frac{R(1+R)^{3}}{60}+\frac{e(1+R)^{3}}{600}+0.15 .
$$

It is not difficult to check that $\frac{e M(R)}{e-1}<R$ if $R \in[0.5,2]$. So the conclusion follows from Corollary 2.4 .

Remark 2.2 Since the coefficient of $x^{3}$ is negative, it appears impossible to find two constants $R>0$ and $\alpha \geq 0$ satisfying (2.12) and (2.13) at the same time.

\section{Existence results for "non-periodic" conditions}

In this section we study the problem of existence of solutions for BVP (1.2).

Lemma 3.1. The BVP (1.2) is equivalent to the integral equation

$$
x(t)=\int_{0}^{t} f(s, x(s),(K x)(s)) \mathrm{d} s-(A+B)^{-1} B \int_{0}^{1} f(s, x(s),(K x)(s)) \mathrm{d} s, \quad t \in[0,1] .
$$

Proof. The result can be obtained by direct computation.

Theorem 3.1. Assume $\operatorname{det} B \neq 0$ and $\left\|B^{-1} A\right\| \leq 1$. Suppose there exist constants $R$ $>0, \alpha \geq 0$ such that

$$
\left(1+\left\|(A+B)^{-1} B\right\|\right) M(R)<R,
$$

and

$$
\begin{gathered}
\|f(t, x,(K x)(t))\| \leq 2 \alpha\langle x, f(t, x,(K x)(t))\rangle+M(R), \\
\forall \lambda \in[0,1] ; \forall(t, x) \in[0,1] \times B_{R}
\end{gathered}
$$

where $M(R)$ is a positive constant depending on $R, B R=\left\{x \in \mathbb{R}^{n},\|x\| \leq R\right\}$. Then $\operatorname{BVP}(1.2)$ has at least one solution $x \in C$ with $\|x\|_{C}<R$.

Proof. Let $C=C\left([0,1], R^{n}\right)$ and $\Omega=\left\{x(t) \in C,\|x(t)\|_{C}<R g\right.$. Define an operator $T: \bar{\Omega} \rightarrow C$ by

$$
T x(t)=\int_{0}^{t} f(s, x(s),(K x)(s)) \mathrm{d} s-(A+B)^{-1} B \int_{0}^{1} f(s, x(s),(K x)(s)) \mathrm{d} s, \quad t \in[0,1](3.3)
$$

Since $f$ is continuous, we see that $T$ is also a continuous map. It is easy to verify that the operator $T$ is compact by the Arzela-Ascoli theorem. It is sufficient to prove

$$
x \neq \lambda T x \text { for all } x \in C \text { with }\|x\|_{C}=R \text { and for all } \lambda \in[0,1] .
$$

See that the family of problems

$$
x=\lambda T x, \lambda \in[0,1]
$$

is equivalent to the family of BVPs

$$
\left\{\begin{array}{l}
x^{\prime}=\lambda f(t, x,(K x)(t)), t \in[0,1] \\
A x(0)+B x(1)=\theta
\end{array}\right.
$$

Consider function $r(t)=\|x(t)\|^{2}, t \in[0,1]$, where $x(t)$ is a solution of (3.6). By the product rule we have 


$$
r^{\prime}(t)=2\left\langle x(t), x^{\prime}(t)\right\rangle=2\langle x(t), \lambda f(t, x(t),(K x)(t))\rangle, t \in[0,1] .
$$

Note that $\left\|B^{-1} A\right\| \| \leq 1$ implies

$$
\|x(1)\|=\left\|B^{-1} A x(0)\right\| \leq\left\|B^{-1} A\right\| \cdot\|x(0)\| \leq\|x(0)\| .
$$

Let $x$ be a solution of (3.5) with $x \in \bar{\Omega}$. We now show that $x \notin \partial \Omega$. From (3.2) and (3.3) we obtain, for each $t \in[0,1]$ and each $\lambda \in[0,1]$,

$$
\begin{aligned}
\|x(t)\| & =\|\lambda T x(t)\| \\
& =\left\|\int_{0}^{t} \lambda f(s, x(s),(K x)(s)) \mathrm{d} s-(A+B)^{-1} B \int_{0}^{1} \lambda f(s, x(s),(K x)(s)) \mathrm{d} s\right\| \\
& \leq\left(1+\left\|(A+B)^{-1} B\right\|\right) \int_{0}^{1} \lambda\|f(s, x(s),(K x)(s))\| \mathrm{d} s \\
& \leq\left(1+\left\|(A+B)^{-1} B\right\|\right) \int_{0}^{1}\|f(s, x(s),(K x)(s))\| \mathrm{d} s \\
& \leq\left(1+\left\|(A+B)^{-1} B\right\|\right) \int_{0}^{1}[2 \alpha\langle x, f(s, x(s),(K x)(s))\rangle+M(R)] \mathrm{d} s \\
& \leq\left(1+\left\|(A+B)^{-1} B\right\|\right) \int_{0}^{1}\left[\alpha \frac{d}{\mathrm{~d} s}\left(\|x(s)\|^{2}\right)+M(R)\right] \mathrm{d} q \\
& \leq\left(1+\left\|(A+B)^{-1} B\right\|\right)\left[\alpha\left(\|x(1)\|^{2}-\|x(0)\|^{2}\right)+M(R)\right] \\
& \leq\left(1+\left\|(A+B)^{-1} B\right\|\right) M(R) .
\end{aligned}
$$

Then it follows from (3.1) that $x \notin \partial \Omega$. Thus, (3.4) is true and the proof is completed.

Corollary 3.1 Let $f$ be a scalar-valued function in (1.1). and assume there exist constants $R>0, \alpha \geq 0$ such that

$$
\frac{3}{2} M(R)<R
$$

and

$$
\begin{gathered}
|f(t, x,(K x)(t))| \leq 2 \alpha\langle x, f(t, x,(K x)(t))\rangle+M(R), \\
\forall \lambda \in[0,1] ; \forall(t, x) \in[0,1] \times B_{R}
\end{gathered}
$$

where $M(R)$ is a positive constant depending on $R, B_{R}=\left\{x \in \mathbb{R}^{n},|x| \leq R\right\}$. Then antiperiodic boundary value problem

$$
\left\{\begin{array}{l}
x^{\prime}=f(t, x,(K x)(t)), t \in[0,1] \\
x(0)=-x(1)
\end{array}\right.
$$

has at least one solution $x \in C[0,1]$ with $|x(t)|<R, t \in[0,1]$.

Proof. Since $A=B=1$, we have $(A+B)^{-1}=\frac{1}{2}, B^{-1} A=1,\left(1+\left\|(A+B)^{-1} B\right\|\right)=\frac{3}{2}$. Then the conclusion follows from Lemma 3.1. 
Example 3.1. Let us show that

$$
\left\{\begin{array}{l}
x^{\prime}=x^{\frac{1}{3}}+x^{3}+\frac{1}{20} \int_{0}^{t} e^{-t s} x(s) \mathrm{d} s+\frac{1}{40} \cos (2 \pi t), \\
x(0)=-x(1) .
\end{array}\right.
$$

has at least one solution $x(t)$ with $|x(t)|<1, \forall t \in[0,1]$.

Denoting $f(t, x,(K x)(t))=x^{\frac{1}{3}}+x^{3}+\frac{1}{20} \int_{0}^{t} \mathrm{e}^{-t s} x(s) \mathrm{d} s+\frac{1}{40} \cos (2 \pi t)$, we see that, for all $(t, x) \in[0,1] \times B_{R}$

$$
|f(t, x,(K x)(t))| \leq|x|^{\frac{1}{3}}+|x|^{3}+\frac{R}{20}+\frac{1}{40} .
$$

On the other hand,

$$
\begin{aligned}
& 2 \alpha\langle x, f(t, x,(K x)(t))\rangle \\
& =2 \alpha\left(x^{\frac{4}{3}}+x^{4}+\frac{x}{20} \int_{0}^{t} e^{-t s} x(s) \mathrm{d} s+\frac{x}{40} \cos (2 \pi t)\right. \\
& =2 x^{\frac{4}{3}}+2 x^{4}+\frac{x}{10} \int_{0}^{t} e^{-t s} x(s) \mathrm{d} s+\frac{x}{20} \cos (2 \pi t), \text { for } \alpha=1 \\
& \geq 2 x^{\frac{4}{3}}+2 x^{4}-\frac{R^{2}}{10}-\frac{R}{20} .
\end{aligned}
$$

Since

$$
\min _{x \in \mathbb{R}}\left\{x^{\frac{4}{3}}+2 x^{4}-|x|^{\frac{1}{3}}-|x|^{3}\right\} \geq-0.4
$$

we choose

$$
M(R)=\frac{R^{2}}{10}+\frac{R}{10}+0.425 .
$$

Then

$$
\|f(t, x,(K x)(t))\| \leq 2\langle x, f(t, x,(K x)(t))\rangle+M(R) .
$$

It is not difficult to check that $\frac{3}{2} M(1)<1$. So, the conclusion follows from Corollary 3.1.

Now we modify Theorem 3.1 to include another class of $f$.

Theorem 3.2. Assume $\operatorname{det} B \neq 0$ and $\left\|A^{-1} B\right\| \leq 1$. Suppose there exist constants $R$ $>0, \alpha \geq 0$ such that

$$
\left(1+\left\|(A+B)^{-1} B\right\|\right) M(R)<R,
$$

and

$$
\begin{gathered}
\|f(t, x,(K x)(t))\| \leq-2 \alpha\langle x, f(t, x,(K x)(t))\rangle+M(R), \\
\forall \lambda \in[0,1] ; \forall(t, x) \in[0,1] \times B_{R}
\end{gathered}
$$

where $M(R)$ is a positive constant depending on $R, B_{R}=\left\{x \in \mathbb{R}^{n},\|x\| \leq R\right\}$. Then BVP (1.2) has at least one solution $x \in C$ with $\|x\|_{C}<R$. 
Proof. Note that $\left\|A^{-1} B\right\| \leq 1$ implies

$$
\|x(0)\|=\left\|A^{-1} B x(1)\right\| \leq\left\|A^{-1} B\right\| \cdot\|x(1)\| \leq\|x(1)\| .
$$

Introducing the function $r(t)=-\|x(t)\|^{2}, t \in[0,1]$, where $x(t)$ is a solution of (3.6), for the rest part of the proof we proceed as in the proof of Theorem 3.1.

Corollary 3.2 Let $f$ be a scalar-valued function in (1.1). If there exist constants $R>0$, $\alpha \geq 0$ such that

$$
\frac{3}{2} M(R)<R
$$

and

$$
\begin{gathered}
|f(t, x,(K x)(t))| \leq-2 \alpha\langle x, f(t, x,(K x)(t))\rangle+M(R), \\
\forall \lambda \in[0,1] ; \forall(t, x) \in[0,1] \times B_{R},
\end{gathered}
$$

where $M(R)$ is a positive constant dependent on $R, B_{R}=\left\{x \in \mathbb{R}^{n},|x| \leq R\right\}$. Then antiperiodic boundary value problem

$$
\left\{\begin{array}{l}
x^{\prime}=f(t, x,(K x)(t)), t \in[0,1] \\
x(0)=-x(1)
\end{array}\right.
$$

has at least one solution $x \in C[0,1]$ with $|x(t)|<R, t \in[0,1]$.

Proof. Since $A=B=1$, we have $(A+B)^{-1}=\frac{1}{2}, A^{-1} B=1,\left(1+\left\|(A+B)^{-1} B\right\|\right)=\frac{3}{2}$. Then the conclusion follows from Lemma 3.2.

In what follows, we discuss the problem of existence of solutions for (1.2) with $f$ satisfying

$$
\|f(t, u, v)\| \leq p(t)\|u\|+q(t)\|v\|+r(t), \quad \forall t \in[0,1], \quad \forall(u, v) \in \mathbb{R}^{n} \times \mathbb{R}^{n},
$$

where nonnegative functions $p, q, r \in L^{1}[0,1]$. We denote $\|x\|_{1}=\int_{0}^{1}|x(t)| \mathrm{d} t$ for any function $x \in L_{1}[0,1]$.

Theorem 3.3. Assume (*) is true and

$$
\left(1+\left\|(A+B)^{-1} B\right\|\right)\left(\|p\|_{1}+K_{0}\|q\|_{1}\right)<1,
$$

where $K_{0}=\max _{0 \leq s \leq t \leq 1}\left\{k_{i}(t, s), i=1,2, \ldots, n\right\}$. Then (1.2) has at least one solution.

Proof. Let $C=C\left([0,1], R^{n}\right)$. Define an operator $T: C \rightarrow C$ by

$$
T x(t)=\int_{0}^{t} f(s, x(s),(K x)(s)) \mathrm{d} s-(A+B)^{-1} B \int_{0}^{1} f(s, x(s),(K x)(s)) \mathrm{d} s, \quad t \in[0,1] .
$$

As we discussed in the proof of Theorem 3.1, $T$ is compact. Taking into account that the family of BVP (1.2) is equivalent to the family of problem $x=T x$, our problem is reduced to show that $T$ has a least one fixed point. For this purpose, we apply Schaefer's Theorem by showing that all potential solutions of

$$
x=\lambda T x, \quad \lambda \in[0,1],
$$

are bounded a priori, with the bound being independent of $\lambda$. With this in mind, let $x$ be a solution of (3.14). Note that $x$ is also a solution of (3.6). We have, for $\forall t \in[0$, $1]$ and $\forall \lambda[0,1]$, 


$$
\begin{aligned}
\|x(t)\| & =\|\lambda T x(t)\| \\
& =\left\|\int_{0}^{t} \lambda f(s, x(s),(K x)(s)) \mathrm{d} s-(A+B)^{-1} B \int_{0}^{1} \lambda f(s, x(s),(K x)(s)) \mathrm{d} s\right\| \\
& \leq\left(1+\left\|(A+B)^{-1} B\right\|\right) \int_{0}^{1} \lambda\|f(s, x(s),(K x)(s))\| \mathrm{d} s \\
& \leq\left(1+\left\|(A+B)^{-1} B\right\|\right) \int_{0}^{1}\|f(s, x(s),(K x)(s))\| \mathrm{d} s \\
& \leq\left(1+\|(A+B)^{-1} B||\right) \int_{0}^{1}[p(t)\|x(s)\|+q(t)\|K x(s)\|+r(s)] \mathrm{d} s \\
& \leq\left(1+\left\|(A+B)^{-1} B\right\|\right)\left[\left(\|p\|_{1}+K_{0}\|q\|_{1}\right)\|x\|_{C}+\|r\|_{1}\right] .
\end{aligned}
$$

Thus,

$$
\|x\|_{C} \leq\left(1+\left\|(A+B)^{-1} B\right\|\right)\left[\left(\|p\|_{1}+K_{0}\|q\|_{1}\right)\|x\|_{C}+\|r\|_{1}\right] .
$$

It then follows from (3.13) that

$$
\|x\|_{C} \leq \frac{\left(1+\left\|(A+B)^{-1} B\right\|\right)\|r\|_{1}}{1-\left[\left(1+\left\|(A+B)^{-1} B\right\|\right)\left(\|p\|\left\|_{1}+K_{0}\right\| q \|_{1}\right)\right]} .
$$

The proof is completed.

Remark 3.1. If $A=B=I$, then (2.13) reduces to

$$
\frac{\sqrt{n}+2}{2}\left(\|p\|_{1}+K_{0}\|q\|_{1}\right)<1 \text {. }
$$

We can also extend the discussion to the existence of at least one solution for integro-differential equations of mixed type with "anti-periodic" conditions.

$$
\left\{\begin{array}{l}
x^{\prime}=f(t, x,(K x)(t),(L x)(t)), t \in[0,1] \\
x(0)=-x(1)
\end{array}\right.
$$

We omit it here because it is trivial.

\section{Acknowledgements}

Research is supported by National Natural Science Foundation of China (10971139), Shanghai municipal education commission(No. 10YZ72)and Shanghai municipal education commission(No. 09YZ149).

\section{Competing interests}

The authors declare that they have no competing interests, All authors read and approved the final manuscript.

Received: 7 December 2010 Accepted: 22 June 2011 Published: 22 June 2011

\section{References}

1. Corduneanu, C: Integral Equations and Applications. Cambridge University Press, Cambridge (1991)

2. Guo, D, Lakshmikantham, V, Liu, X: Nonlinear Integral Equations in Abstract Spaces. Kluwer Academic, Dordrecht (1996)

3. Agarwal, R, Bohner, M, Domoshnitsky, A, Goltser, Y: Floquet theory and stability of nonlinear integro-differential equations. Acta Math Hungar. 109, 305-330 (2005). doi:10.1007/s10474-005-0250-7

4. Franco, D: Green's functions and comparison results for impulsive integro-differential eqations. Nonlinear Anal. 47, 5723-5728 (2001). doi:10.1016/50362-546X(01)00674-5

5. Guo, D: Initial value problems for integro-differential equaitons of Volterra type in Banach spaces. J Appl Math Stochastic Anal. 7, 13-23 (1994). doi:10.1155/S104895339400002X 
6. He, Z, He, X: Monotone iterative technique for impulsive integro-differential equations with periodic boundary conditions. Comput Math Appl. 48, 73-84 (2004). doi:10.1016/j.camwa.2004.01.005

7. He, Z, He, X: Periodic boundary value problems for first order impulsive integro-differential equations of mixed type. J Math Anal Appl. 296, 8-20 (2004). doi:10.1016/j.jmaa.2003.12.047

8. Jankowski, T, Jankowski, R: On integro-differential equations with delayed arguments. Dyn Continuous Discrete Impuls Syst Ser A Math Anal. 13, 101-115 (2006)

9. Ladde, GS, Sathanantham, S: Periodic boundary value problem for impulsive integro-differential equations of Volterra type. J Math Phys Sci. 25, 119-129 (1991)

10. Li, J, Shen, J: Periodic boundary value problems for impulsive integro-differential equations of mixed type. Appl Math Comput. 183, 890-902 (2006). doi:10.1016/j.amc.2006.06.037

11. Nieto, JJ, Rodriguez Lopez, R: Periodic boundary value problems for non-Lipschitzian impulsive functional differential equations. J Math Anal Appl. 318, 593-610 (2006). doi:10.1016/j.jmaa.2005.06.014

12. Song, G: Initial value problems for systems of integro-differential equations in Banach spaces. J Math Anal Appl. 264, 68-75 (2001). doi:10.1006/jmaa.2001.7630

13. Song, $G, Z$ Zhu, X: Extremal solutions of periodic boundary value problems for first order integro-differential equations of mixed type. J Math Anal Appl. 300, 1-11 (2004). doi:10.1016/j.jmaa.2004.02.060

14. Xing, $Y$, Han, $M$, Zheng, G: Initial value problem for first order integro-differential equation of Volterra type on time scales. Nonlinear Anal. 60, 429-442 (2005)

15. Xing, Y, Ding, W, Han, M: Periodic boundary value problems of integro-differential equations of Volterra type on time scales. Nonlinear Anal. 68, 127-138 (2008). doi:10.1016/..na.2006.10.036

16. $\mathrm{Xu}, \mathrm{H}$, Nieto, JJ: Extremal solutions of a class of nonlinear integro-differential equations in Banach spaces. Proc Am Math Soc. 125, 2605-2614 (1997). doi:10.1090/50002-9939-97-04149-X

17. Zhang, X, Jiang, D, Li, X, Wang, K: A new existence theory for single and multiple positive periodic solutions to Volterra integro-differential equations with impulse effects. Comput Math Appl. 51, 17-32 (2006). doi:10.1016/j. camwa.2005.09.002

18. Tisdell, CC: Existence of solutions to first -order periodic boundary value problems. J Math Anal Appl. 323(2), 1325-1332 (2006). doi:10.1016/j.jmaa.2005.11.047

19. Tisdell, CC: On first-order discrete boundary value problems. J Diff Equ Appl. 12, 1213-1223 (2006). doi:10.1080/ 10236190600949790

20. Chen, Y, Nieto, JJ, O'Regan, D: Anti-periodic solutions for fully nonlinear first-order differential equations. Math Comput Modell. 46(9-10), 1183-1190 (2007). doi:10.1016/j.mcm.2006.12.006

21. Ding, $W$, Xing, $Y$, Han, M: Anti-periodic boundary value problems for first order impulsive functional differential equations. Appl Math Comput. 186, 45-53 (2007). doi:10.1016/j.amc.2006.07.087

22. Franco, D, Nieto, JJ, O'Regan, D: Anti-periodic boundary value problem for nonlinear first order ordinary differential equations. Math Inequal Appl. 6, 477-485 (2003)

23. Luo, ZG, Shen, JH, Nieto, JJ: Antiperiodic boundary value problem for first -order impulsive ordinary differential equations. Comput Math Appl. 49, 253-261 (2005). doi:10.1016/j.camwa.2004.08.010

24. Skóra, L: Monotone iterative method for differential systems with impulses and anti-periodic boundary condition. Comment Math Prace Mat. 42(2), 237-249 (2002)

25. Lloyd, NG: Degree Theory. Cambridge University Press, Cambridge, New York, Melbourne (1978)

doi:10.1186/1687-1847-2011-14

Cite this article as: Xing and Fu: Some new results for BVPs of first-order nonlinear integro-differential equations

of volterra type. Advances in Difference Equations 2011 2011:14

\section{Submit your manuscript to a SpringerOpen ${ }^{\odot}$ journal and benefit from:}

- Convenient online submission

- Rigorous peer review

- Immediate publication on acceptance

- Open access: articles freely available online

- High visibility within the field

- Retaining the copyright to your article

Submit your next manuscript at $\boldsymbol{s p r i n g e r o p e n . c o m ~}$ 